\title{
Ulinastatin inhibits apoptosis induced by serum deprivation in mesenchymal stem cells
}

\author{
LINHUI SHI, LONGQIANG YE, PANPAN LIU, DANQIN LIU, \\ GONGJIE YE, JIAHONG CHEN and ZHOUZHOU DONG \\ Critical Care Unit, Ningbo Medical Center Lihuili Eastern Hospital, \\ Taipei Medical University Ningbo Medical Center, Ningbo, Zhejiang 315040, P.R. China
}

Received December 22, 2017; Accepted October 10, 2018

DOI: $10.3892 / \mathrm{mmr} .2019 .9847$

\begin{abstract}
Mesenchymal stem cells (MSCs) have exhibited great potential in the therapy of cardiovascular disease. However, the application of MSCs is hampered by apoptosis, which reduces the number of cells in the host cardiac microenvironment. Ulinastatin (UTI), a broad-spectrum protease inhibitor that can be purified from human urine, has attracted attention for its protective effects through its immunomodulatory and anti-inflammatory properties. The present study aimed to evaluate the effects of UTI on serum deprivation-induced apoptosis of MSCs and investigate its molecular mechanisms. Cell viability was determined by the MTT assay. Apoptosis was assessed by flow cytometric analysis with Annexin V/propidium iodide staining. The protein levels of cleaved caspase-3, B-cell lymphoma-2 (Bcl-2) family proteins, total-Akt and phospho-Akt were evaluated by western blot. The results of the present study demonstrated that UTI exhibited a protective effect in serum deprived MSCs, as indicated by increased cell viability, and a reduction in the rate of apoptosis and caspase- 3 activation. In addition, treatment with UTI significantly decreased the expression levels of Bcl-2, Bcl-extra large and Bcl-associated $\mathrm{X}$ protein. Furthermore, activation of the Akt signaling pathway was involved in the UTI-induced anti-apoptotic effects. The present findings indicated that UTI is able to promote the survival of MSCs under serum deprivation conditions. The present study may be helpful in improving the therapeutic efficacy of MSC transplantation used to cure chronic ischemic heart disease.
\end{abstract}

Correspondence to: Dr Zhouzhou Dong, Critical Care Unit, Ningbo Medical Center Lihuili Eastern Hospital, Taipei Medical University Ningbo Medical Center, 1111 Jiangnan Road, Ningbo, Zhejiang 315040, P.R. China

E-mail: dongzhouzhou2010@163.com

Key words: ulinastatin, mesenchymal stem cells, apoptosis, Akt serine/threonine kinase

\section{Introduction}

Although there has been great improvement in the treatment of heart failure, ischemia/reperfusion (I/R) injury remains a leading cause of mortality in Western countries (1). Numerous studies have indicated that transplantation of mesenchymal stem cells (MSCs) represents a potential tool for the repair and regeneration of cardiomyocytes, thus restoring the function of the heart (2). However, the effects of MSCs are limited because the majority of transplanted MSCs undergo cell death soon after transplantation in the ischemic microenvironment (3). Thus, great efforts have been made to improve the survival of donor MSCs. The lack of cellular growth factors and/or insufficient blood supply to the ischemic region are considered to be the key factors contributing to the high rate of MSC transplantation failure (4). Therefore, hypoxia and serum deprivation (H/SD) conditions can be used to mimic the hostile ischemic microenvironment (4). The enhancement of MSC viability and survival under ischemic conditions may be a strategy to improve the efficiency of MSC therapy.

Ulinastatin (UTI), a broad-spectrum protease inhibitor that can be purified from human urine, has attracted attention for its protective effects via immunomodulatory and anti-inflammatory properties. For instance, it has been reported that UTI protects human endothelial cells from oxidative damage by suppressing the c-Jun $\mathrm{N}$-terminal kinase/c-Jun signaling pathway (5). UTI was also reported to ameliorate I/R injury in the spinal cord (6). Furthermore, a recent study showed that UTI protected against lipopolysaccharide-induced cardiac microvascular endothelial cell dysfunction (7). However, the protective effects of UTI on MSCs for regeneration have not been investigated yet. In the current study, it was hypothesized that UTI may be able to repress apoptosis induced by H/SD conditions and thereby improve the survival of MSCs. To confirm this, the effects of UTI on the H/SD-induced apoptosis of MSCs and the underlying molecular mechanisms were investigated.

\section{Materials and methods}

Materials. UTI was purchased from Beijing Solarbio Science \& Technology Co., Ltd. (Beijing, China); antibodies for caspase-3 (cat. no. 14220S), B-cell lymphoma (Bcl)-2 
(cat. no. 15071S), induced myeloid leukemia cell differentiation protein Mcl-1 (Mcl-1; cat. no. 94296S), Bcl-extra large (Bcl-xl; cat. no. 2762S), Bcl-associated protein X (Bax; cat. no. 2772S), activating transcription factor 4 (ATF4; cat. no. 11815S), GAPDH (cat. no. 8884S), horseradish peroxidase-linked secondary anti-rabbit (cat. no. 7074) and anti-mouse (cat. no. 7076) were obtained from Cell Signaling Technology, Inc. (Danvers, MA, USA). C/EBP homologous protein (CHOP; cat. no. ab11419), phospho-Akt (cat. no. ab38449), total Akt (cat. no. ab85683), phospho-phosphoinositide 3-kinase (PI3K; cat. no. ab182651), total-PI3K (cat. no. ab32089), phospho-mammalian target of rapamycin (mTOR; ab109268) and total-mTOR (cat. no. ab134903) were purchased from Abcam (Cambridge, UK). The Annexin V-fluorescein isothiocyanate (FITC) Apoptosis Detection Kit was purchased from BD Bioscience (Franklin Lakes, NJ, USA). The Caspase-3 Activity Colorimetric Assay Kit was purchased from Beyotime Institute of Biotechnology (Haimen, China). LY294002 and wortmannin, which were used at a concentration of $20 \mu \mathrm{M}$, were purchased from Selleck Chemicals (Houston, TX, USA). Cells were pretreated with LY294002 and wortmannin for $1 \mathrm{~h}$ at $37^{\circ} \mathrm{C}$ prior to experimentation. All other chemicals were obtained from Sigma-Aldrich (Merck KGaA, Darmstadt, Germany). Rapamycin was purchased from Sigma-Aldrich (Merck $\mathrm{KGaA}$ ), which was kept as $1 \mathrm{mM}$ stock at $-80^{\circ} \mathrm{C}$ and used at $20 \mu \mathrm{M}$. Cells were pretreated with rapamycin for $1 \mathrm{~h}$ at $37^{\circ} \mathrm{C}$ prior to experimentation.

Culture of MSCs. MSCs were isolated from the bone marrow of two male 2-week-old Sprague-Dawley rats (weighing 60-80 g) as described earlier (8). The rats were maintained under the following conditions: Temperature, $22 \pm 2{ }^{\circ} \mathrm{C}$; humidity, 55 $5 \%$; 12-h light/dark cycle; free access to food and water. All studies were performed under the approval of the Institutional Animal Care and Use Committee of Ningbo Medical Center Lihuili Eastern Hospital (Ningbo, China). Briefly, MSCs were flushed from femurs and tibias with $10 \mathrm{ml}$ Dulbecco's-modified Eagle's medium (DMEM; Gibco; Thermo Fisher Scientific, Inc., Waltham, MA, USA) containing $1 \%$ penicillin/streptomycin (HyClone; GE Healthcare, Logan, UT, USA). The cells were centrifuged at $300 \mathrm{x} \mathrm{g}$ for $5 \mathrm{~min}$ at $4^{\circ} \mathrm{C}$. The pellets were resuspended in $6 \mathrm{ml}$ DMEM with $10 \%$ fetal bovine serum (HyClone; GE Healthcare) and $1 \%$ penicillin/streptomycin and plated in a plastic flask at $37^{\circ} \mathrm{C}$ in a humidified atmosphere containing $5 \% \mathrm{CO}_{2}$. After 3 days, the medium was replaced, and the cells were washed with PBS. The medium was replaced every 3 days. At 8 days after seeding, the cells became $>70 \%$ confluent. Subsequently, the cells were collected from the dishes and expanded at a 1:2 dilution. All subsequent experiments were performed using MSCs at passages 3-5.

For the identification of the MSC immunophenotypic characteristics, the cells were collected, washed with PBS (HyClone; GE Healthcare) and labeled with the following antibodies: Phycoerythrin (PE)-labeled anti-cluster of differentiation 45 (CD45; cat. no. 553091; BD Pharmingen; BD Biosciences), anti-CD105 (cat. no. 611314; BD Pharmingen; BD Biosciences), anti-CD90 (cat. no. 55595; BD Pharmingen; BD Biosciences) and FITC-labeled-CD73 (cat. no. 561254; BD Pharmingen; BD Biosciences). Subsequently, the cells were detected by flow cytometry and analyzed using FACSDiva software (version 6.1.3; BD Biosciences).

Small interfering RNA (siRNA) transfection. MSCs $\left(4 \times 10^{5}\right.$ cells/well) were seeded in 6 -well plates. When cells reached $\sim 70 \%$ confluence, MSCs were transfected with 200 pM negative control siRNA (5'-ACGGAACAGCGCACC GAGGCGAA-3'), siRNA against CHOP (5'-CCAGGAAAC GGAAACAGAGTT-3') or ATF4 (5'-TCCCTCCATGTG TAAAGGA-3'; Genepharm Inc., Sunnyvale, CA, USA) using Lipofectamine ${ }^{\circledR} 2000$ (Invitrogen; Thermo Fisher Scientific, Inc.) according to the manufacturer's protocol. A total of $24 \mathrm{~h}$ post-transfection, cells were harvested and the effects of knockdown were investigated.

H/SD treatment. The harvested MSCs were cultured in 6-well ( $1 \times 10^{5}$ cells/well) or 96 -well plates $\left(1 \times 10^{4}\right.$ cells/well) (Corning Incorporated, Corning, NY, USA) for subsequent experiments. To induce MSC apoptosis by exposing them to H/SD conditions, the normal medium was replaced with glucose- and serum-free medium. Subsequently, the MSCs were placed in an oxygen-free incubator for $12 \mathrm{~h}$. MSCs cultured under normal conditions were used as controls.

MTT assay. Cell viability was assayed using the MTT assay as described previously (9). Briefly, cells were seeded in 96-well plates with a density of $2 \times 10^{4} /$ well. When reaching $~ 80 \%$ confluence, the cells were exposed to H/SD treatment with or without UTI (100, 200 and $400 \mathrm{U} / \mathrm{ml})$. After $12 \mathrm{~h}, 25 \mu \mathrm{l}$ MTT ( $5 \mathrm{mg} / \mathrm{ml}$; Sigma-Aldrich; Merck KGaA) was added to the cultured media, and the cells were incubated for another $4 \mathrm{~h}$. The medium was then removed, and $200 \mu$ l dimethyl sufoxide was added to each well. The absorbance of the solutions was measured using a microplate reader (BioTek China, Beijing, China) at $495 \mathrm{~nm}$. The relative cell viability was measured by comparison with cells cultured under normal conditions. The calculated cell viability percentages from the three parallel experiments were averaged for each set of experimental conditions.

Apoptosis analysis. Cellular apoptosis was detected using an Annexin V/propidium iodide (PI) Apoptosis Detection kit (BD Pharmingen; BD Biosciences) according to the manufacturer's protocol. Briefly, $1 \times 10^{5}$ cells were harvested and washed in ice-cold PBS, then cells were resuspended in $300 \mu \mathrm{l}$ binding buffer and incubated with $5 \mu 1$ Annexin V-FITC solution for $30 \mathrm{~min}$ in the dark at $4^{\circ} \mathrm{C}$, followed by further incubation with $5 \mu \mathrm{l}$ PI for $5 \mathrm{~min}$ at room temperature. Apoptotic cells were then determined using a flow cytometer (FACSCalibur; BD Biosciences). The results were analyzed by the FlowJo 10.4 (FlowJo LLC, Ashland, OR USA).

Western blot analysis. Cells were collected and lysed in radioimmunoprecipitation buffer (Beyotime Institute of Biotechnology). Equal amounts of lysates $(20 \mu \mathrm{g})$ were resolved by SDS-PAGE on $12 \%$ gels and transferred to polyvinylidene fluoride (PVDF) membranes. PVDF membranes were incubated with primary antibodies at a dilution of 1:1,000 overnight at $4^{\circ} \mathrm{C}$. Subsequently, the membranes were washed three times with TBS with Tween 20 and incubated 
for $1 \mathrm{~h}$ at room temperature with the corresponding horseradish peroxidase-conjugated secondary antibodies (1:10,000; anti-mouse cat. no. 7076, or anti-rabbit cat. no. 7074; Cell Signaling Technology, Inc.). The signals were visualized using an enhanced chemiluminescence reagent (Pierce; Thermo Fisher Scientific, Inc.). The density of the protein bands was analyzed using ImageJ 1.41 software (National Institutes of Health, Bethesda, MD, USA).

Caspase-3 activity assay. The caspase-3 activity was assayed using the Caspase-3 Activity Colorimetric Assay kit (Beyotime Institute of Biotechnology) according to the manufacturer's protocol. In brief, cellular lysates $(150 \mu \mathrm{g})$ were incubated with $50 \mu \mathrm{l}$ reaction buffer containing $10 \mathrm{mM}$ dithiothreitol for $1 \mathrm{~h}$ at $37^{\circ} \mathrm{C}$ in the substrate DEVD-pNA $(200 \mu \mathrm{M})$ in a total volume of $100 \mu \mathrm{l}$. The pNA light emission following cleavage from the substrate was quantified using a microplate reader (BioTek China) at $405 \mathrm{~nm}$.

Statistical analysis. Statistical analysis was performed using SPSS 19.0 software (SPSS, Inc., Chicago, IL, USA). Experiments were repeated three times and data are expressed as the mean \pm standard deviation. Differences among groups were tested by one-way analysis of variance, followed by Tukey's test. $\mathrm{P}<0.05$ was considered to indicate a statistically significant difference.

\section{Results}

UTI promotes the viability of MSCs under H/SD conditions. MSC characteristics were confirmed by flow cytometric analysis, which showed that the cells were negative for CD45, and positive for CD90, CD103 and CD73 markers (data not shown). To investigate the protective effects of UTI, cell viability was initially examined. As shown in Fig. 1A, UTI exhibited little cytotoxicity on MSCs under normal culture conditions. Cells were treated with increasing concentrations of UTI $(100,200$ and $400 \mathrm{U} / \mathrm{ml})$ when exposed to H/SD for $12 \mathrm{~h}$ (Fig. 1B). The data revealed that $\mathrm{H} / \mathrm{SD}$ reduced cell viability and UTI protected MSCs against H/SD injury in a dose-dependent manner.

UTI protects MSCs from apoptosis under H/SD conditions. To further analyze the cytoprotective effects of UTI, the apoptosis rate of the MSCs was assayed by Annexin V/PI staining and flow cytometry. As indicated in Fig. 2A and B, treatment with UTI reduced the apoptosis induced by H/SD in a dose-dependent manner. Subsequently, the levels of cleaved caspase- 3 were evaluated by western blotting. Cleaved caspase-3 was notably inhibited by UTI in a dose-dependent manner (Fig. 2C). Furthermore, caspase-3 activity assays revealed that activation of caspase- 3 induced by H/SD was also decreased by UTI in a dose-dependent manner (Fig. 2D). Taken together, these findings demonstrated that UTI protects MSCs against apoptosis under H/SD conditions.

UTI treatments modulate Bcl-2 family protein expression levels. The process of apoptosis is regulated by $\mathrm{Bcl}-2$ family proteins, which can be divided into anti-apoptotic and pro-apoptotic members (10). Therefore, whether Bcl-2 family members were affected by UTI was investigated. As indicated in Fig. 3, anti-apoptotic Bcl-2, Mcl-1 and Bcl-xl were decreased, while pro-apoptotic Bax was upregulated in MSCs exposed to H/SD conditions. Treatment with UTI reversed the effects of H/SD on Bcl-2 family proteins (Fig. 3). These findings suggested that UTI exerts a protective effect against $\mathrm{H} / \mathrm{SD}$-induced apoptosis via modulation of $\mathrm{Bcl}-2$ family proteins in MSCs.

UTI protects MSCs from H/SD-induced apoptosis via inhibition of endoplasmic reticulum (ER) stress. Previous studies indicated that ATF4 and CHOP are vital players in ER stress that are involved in apoptosis induced by H/SD (11). To determine whether ER stress is involved in the protective effect of UTI, ATF4 and CHOP expression levels were examined by western blotting. As shown in Fig. 4A, H/SD induced the upregulation of ATF4/CHOP, and treatment with UTI reversed the effect of $\mathrm{H} / \mathrm{SD}$ on $\mathrm{ATF} 4 / \mathrm{CHOP}$ expression levels. To further confirm the role of ER stress in H/SD-induced apoptosis of MSCs, siRNA was performed to knock down CHOP and ATF4 expression levels (Fig. 4B). As indicated in Fig. 4C, silencing of CHOP or ATF4 protects MSCs from apoptosis induced by H/SD. Taken together, these results suggested that UTI protects MSCs from H/SD-induced apoptosis, at least partially, by inhibiting ER stress.

Inhibition of PI3K/Akt/mTOR could interfere with the protective effects of UTI against apoptosis induced by $H / S D$. The PI3K/Akt/mTOR signaling pathway serves a critical role in cell survival (9). Thus, to examine whether the PI3K/Akt/mTOR pathway is also involved in the anti-apoptotic effects of UTI, its activation was investigated by western blotting. As shown in Fig. 5A, H/SD treatment reduced expression levels of phospho-Akt, phospho-PI3K and phospho-mTOR compared with the control, whereas these effects were reversed by UTI treatment. To verify that the PI3K/Akt/mTOR signaling pathway was involved in UTI-mediated anti-apoptotic effects, two PI3K inhibitors, wortmannin and LY294002, were used. As shown in Fig. 5B, $\mathrm{H} / \mathrm{SD}$ inhibited the expression of phospho-Akt, which can be activated by UTI treatment. However, both wortmannin $(20 \mu \mathrm{M})$ and LY294002 $(20 \mu \mathrm{M})$ fully inhibited the upregulation of phospho-Akt induced by UTI (Fig. 5B). Furthermore, the apoptotic cells were increased after wortmannin and LY294002 treatment compared with the UTI group under H/SD conditions (Fig. 5C). Additionally, rapamycin, an mTOR inhibitor $(20 \mu \mathrm{M})$, was applied to investigate the role of mTOR in the protective effects of UTI. As indicated in Fig. 5D, rapamycin successfully inhibited the phosphorylation of mTOR. Additionally, the apoptotic cells increased following rapamycin treatment compared with the UTI group under H/SD conditions (Fig. 5E). Taken together, these findings further confirmed that UTI exerts protective effects via activation of the PI3K/Akt/mTOR pathway.

\section{Discussion}

MSCs have exhibited great therapeutic potential due to their ability to regenerate and repair the injured myocardium through paracrine mechanisms following transplantation into 

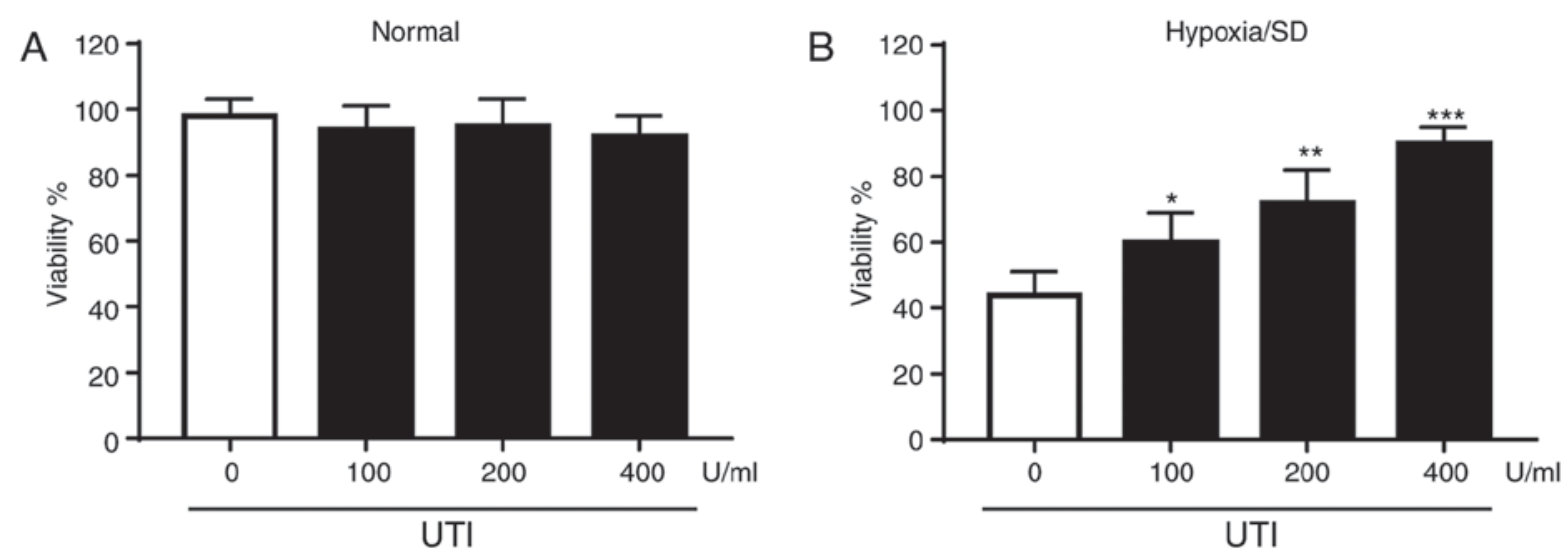

Figure 1. Effects of UTI on the viability of MSCs. (A) MSCs were cultured under normal conditions and (B) under hypoxia/SD conditions with various doses of UTI for $12 \mathrm{~h}$, and cell viability was assayed by MTT assay. The data are presented as the mean \pm standard deviation $(\mathrm{n}=3)$. ${ }^{*} \mathrm{P}<0.05 ;{ }^{* * *} \mathrm{P}<0.01 ;{ }^{* * * *} \mathrm{P}<0.001$, vs. the no UTI. MSC, mesenchymal stem cell; SD, serum deprivation; UTI, ulinastatin.
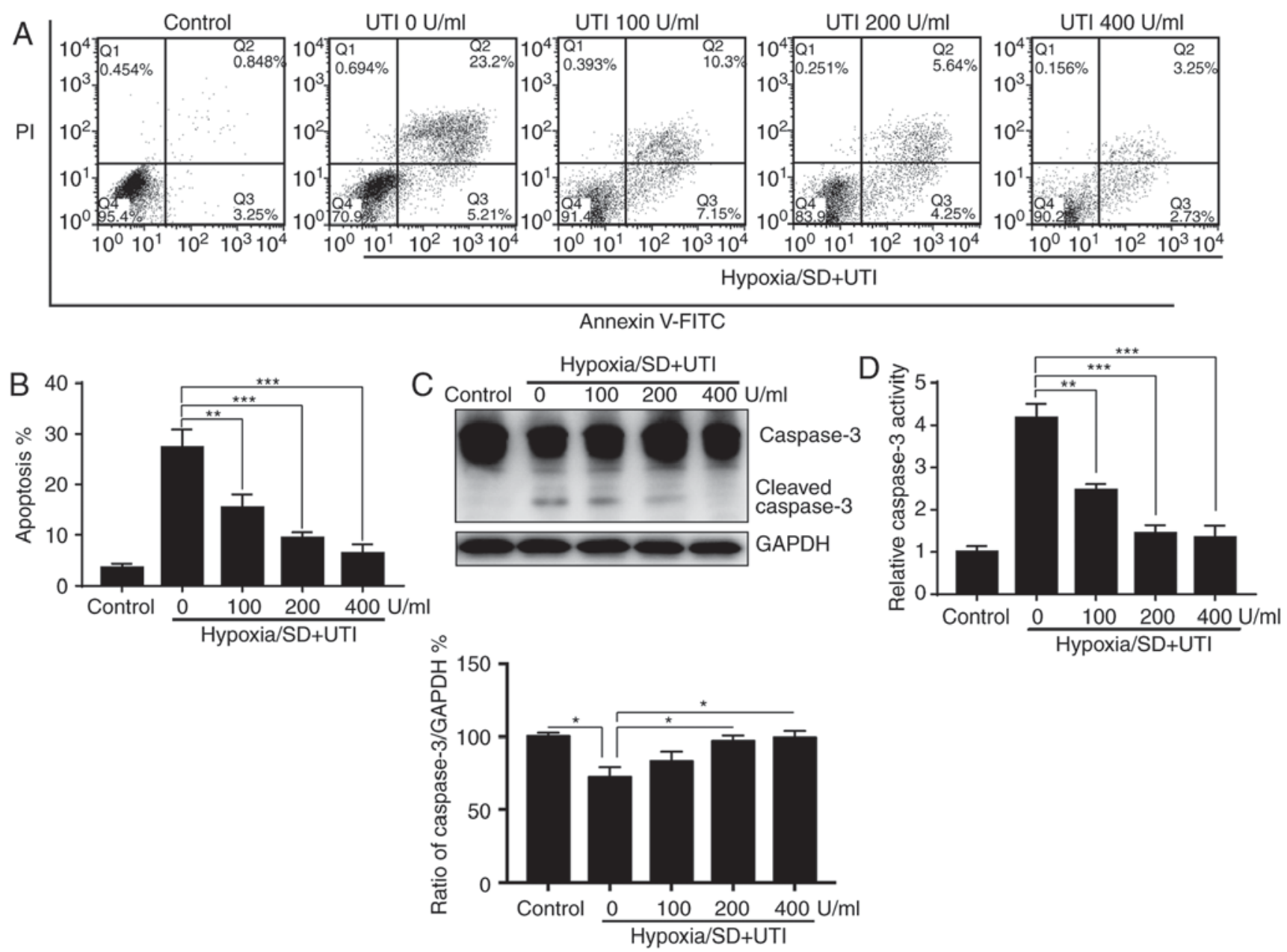

Figure 2. UTI protects MSCs against hypoxia/SD-induced apoptosis. MSCs were treated with various doses of UTI under hypoxia/SD conditions for $12 \mathrm{~h}$. (A) Apoptosis rates were measured using flow cytometry with Annexin V/PI staining and (B) data were quantified. (C) Cellular lysates were subjected to western blot analysis with the indicated antibodies. (D) Caspase-3 activity was determined using a colorimetric assay kit. The data are presented as the mean \pm standard deviation $(\mathrm{n}=3)$. ${ }^{*} \mathrm{P}<0.05,{ }^{* *} \mathrm{P}<0.01,{ }^{* * *} \mathrm{P}<0.001$. MSC, mesenchymal stem cell; UTI, ulinastatin; PI, propidium iodide; SD, serum deprivation; FITC, fluorescein isothiocyanate.

an ischemic or infarcted heart $(12,13)$. Since MSCs are easily obtained and expanded, they also represent one of the cell therapy tools with the most potential for tissue regeneration in various clinical diseases (14). However, the therapeutic application of MSCs is limited by the fact that the majority of the implanted MSCs do not survive transplantation, partially due to cell death induced by the ischemic cardiac microenvironment (15). Hodgetts et al (16) reported that $>90 \%$ of MSCs 

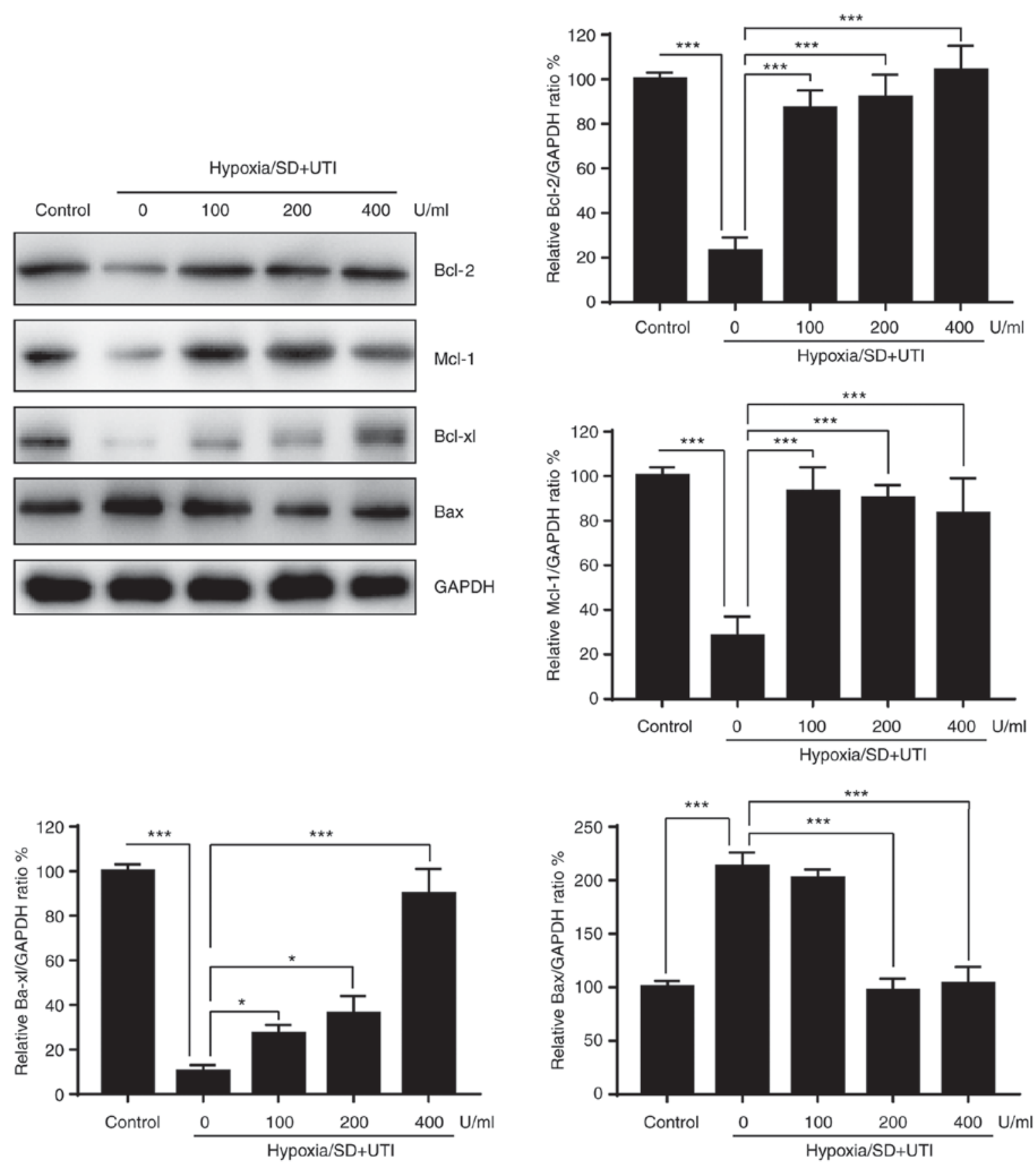

Figure 3. Effects of UTI on the Bcl-2 family proteins. MSCs were treated with various doses of UTI under hypoxia/SD conditions for $12 \mathrm{~h}$. Subsequently, cellular lysates were subjected to western blot analysis with the indicated antibodies, and the results were quantified. The data are presented as the mean \pm standard deviation $(\mathrm{n}=3)$. ${ }^{*} \mathrm{P}<0.05 ;{ }^{* * *} \mathrm{P}<0.001$, vs. the group without UTI. MSC, mesenchymal stem cell; SD, serum deprivation; UTI, ulinastatin; Bcl, B-cell lymphoma; Bax, Bcl-associated X protein; Mcl-1, induced myeloid leukemia cell differentiation protein Mcl-1; Bcl-xl, Bcl-extra large.

die within $24 \mathrm{~h}$ of transplantation. Another study reported that $21 \%$ of MSCs survive after $4 \mathrm{~h}$ of transplantation and only $3.6 \%$ survive after 7 days (17). Therefore, great efforts have been made to prevent stem cell apoptosis to improve their therapeutic potential. One available strategy to enhance MSC survival is treatment with agents that can enhance the viability of MSCs. For example, a previous study reported that prostaglandin E improved MSC survival under H/SD conditions, which is a commonly used model to mimic the ischemic environment in vitro (18). Another study reported that nicorandil protected MSCs from H/SD-induced apoptosis (19). In the present study, UTI inhibited the apoptosis of MSCs in a dose-dependent manner under H/SD conditions.
Furthermore, the anti-apoptotic effects of UTI were revealed to be exerted via the modulation of Bcl-2 family proteins, ER stress and PI3K/Akt signaling pathways. The present study revealed the protective effects of UTI on MSCs under H/SD conditions in vitro, and the mechanisms leading to protection were investigated.

UTI is a multivalent Kunitz-type serine protease inhibitor that can be isolated from human urine and blood (20). UTI has been reported to protect organs against injury via inhibition of various proteases, such as trypsin, chymotrypsin, neutrophil elastase and plasmin (21). Furthermore, UTI also possesses therapeutic potential to treat inflammatory diseases and subsequent organ damage with few side effects (22). 
A
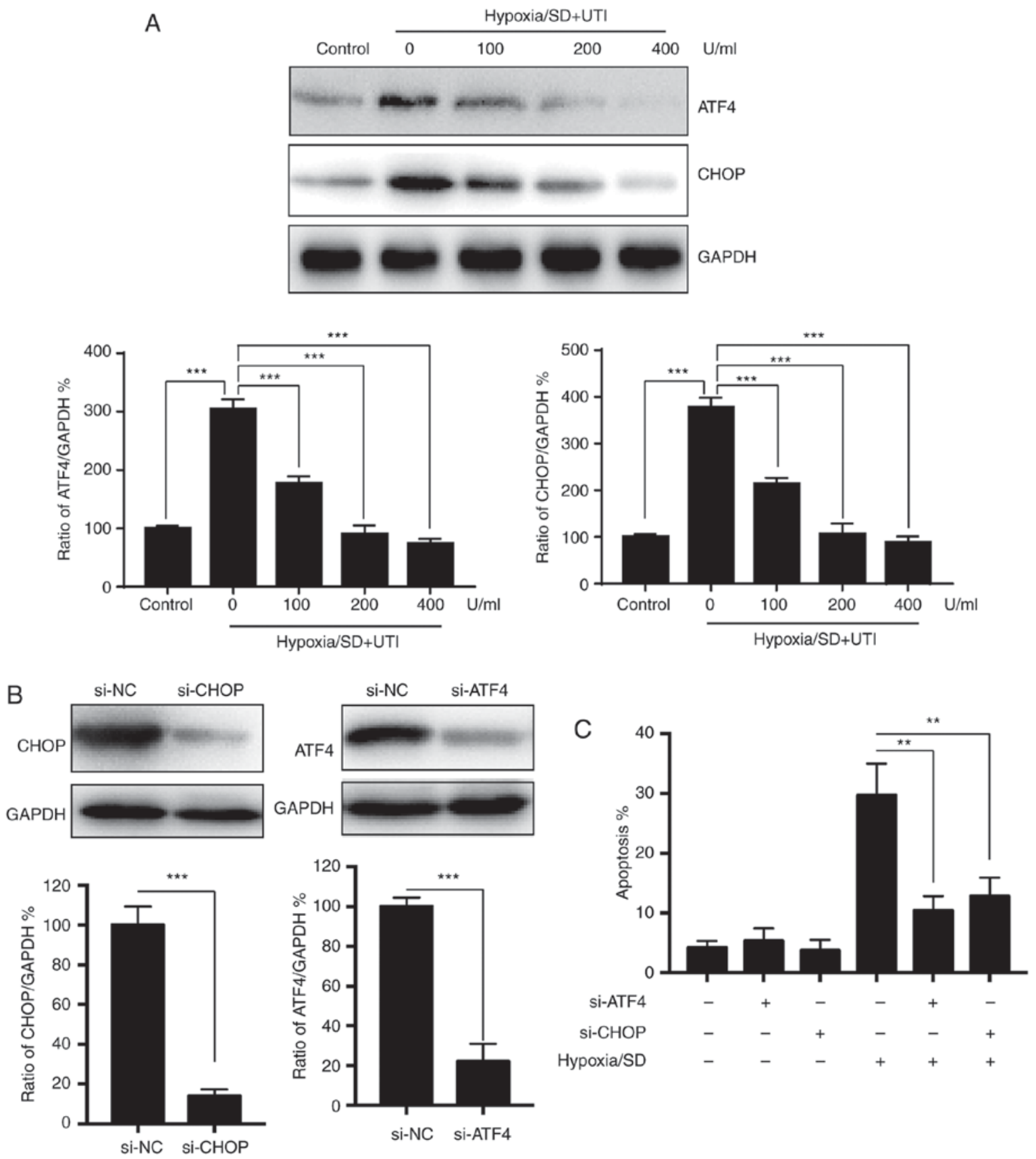

Figure 4. UTI reduces apoptosis in MSCs by inhibiting ER stress. MSCs were treated with various doses of UTI under hypoxia/SD condiions for $12 \mathrm{~h}$. (A) Total cellular lysates were subjected to western blot analysis with the indicated antibodies, and the results were quantified. (B) MSCs were transfected with siRNA against CHOP or ATF4 for $24 \mathrm{~h}$, and the expression of CHOP or ATF4 was detected by western blotting. (C) MSCs were transfected with siRNA against CHOP or ATF4 for $24 \mathrm{~h}$, then cells were cultured under hypoxia/SD conditions or not for another $24 \mathrm{~h}$, and apoptosis was quantified. The data are presented as the mean \pm standard deviation $(\mathrm{n}=3)$. ${ }^{* *} \mathrm{P}<0.01 ;{ }^{* * *} \mathrm{P}<0.001$. MSC, mesenchymal stem cell; SD, serum deprivation; UTI, ulinastatin; ATF4, activating transcription factor 4; CHOP, C/EBP homologous protein; si, small interfering RNA; NC, normal control.

In the present study, UTI enhanced the proliferation of MSCs in a dose-dependent manner. UTI also repressed the apoptosis induced by H/SD and modulated the expression of Bcl-2 family of proteins. Apoptosis is a complex multistep process that is subjected to regulation by various proteins. Apoptosis is predominantly triggered via two pathways: The extrinsic and the intrinsic/mitochondrial pathway (9). Deprivation of serum is a well-established cause of apoptosis via activation of the intrinsic/mitochondrial pathway (23). The intrinsic apoptotic pathway is controlled by Bcl-2 family proteins (10). The effects of UTI on the inhibition of caspase-3 and the regulation of Bcl-2 family proteins indicated that UTI repressed apoptosis via blocking the intrinsic/mitochondrial pathway. These findings were in line with a previous study in which UTI enhanced the expression of Bcl-2, and thereby, inhibited cell apoptosis in an animal model of hemorrhagic shock (24).

The ER is an organelle involved in calcium homeostasis, lipid biosynthesis and protein folding. Various stimuli, such as ischemia, hypoxia, heat shock, and oxidative stress, can lead to ER dysfunction, which is also termed ER stress (25). It has been demonstrated that ER stress serves an important 
A
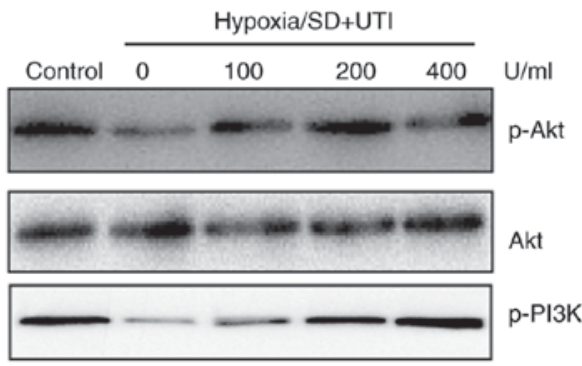

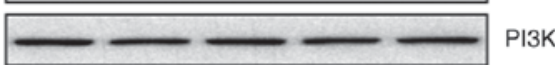
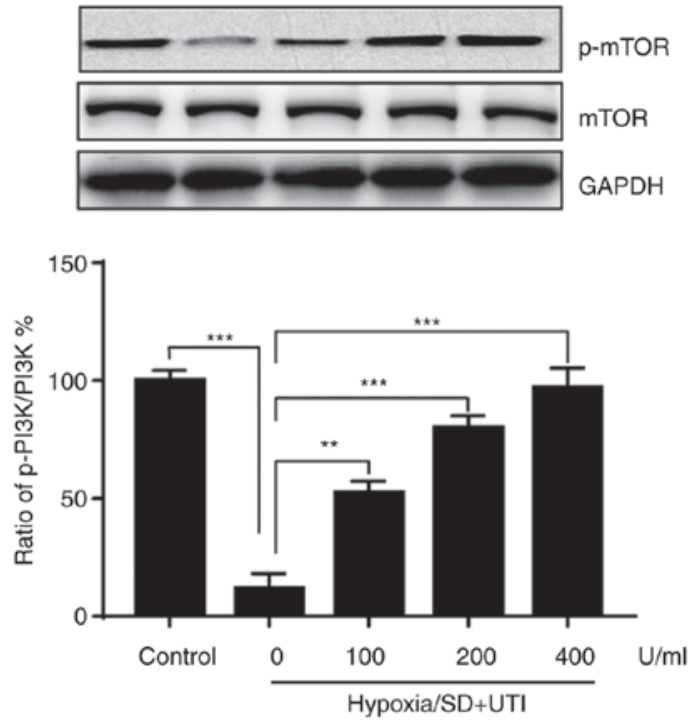

B Hypoxia/SD - ++++++

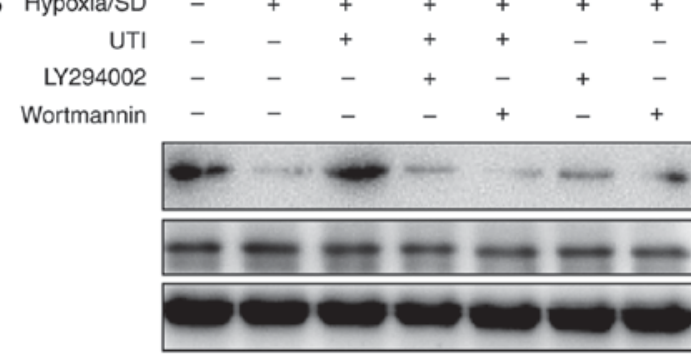

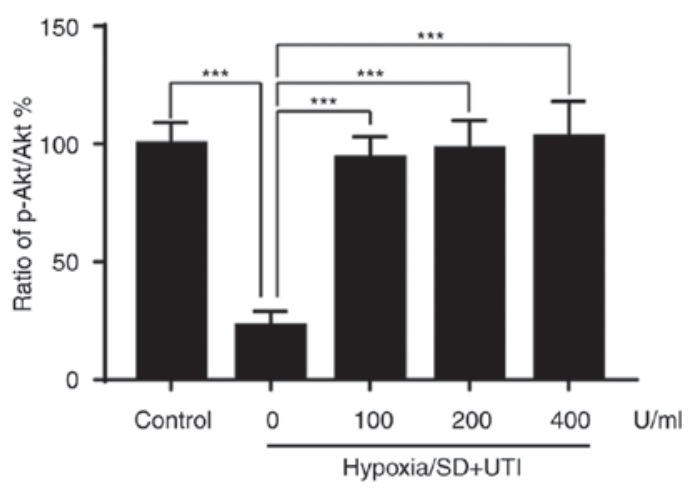

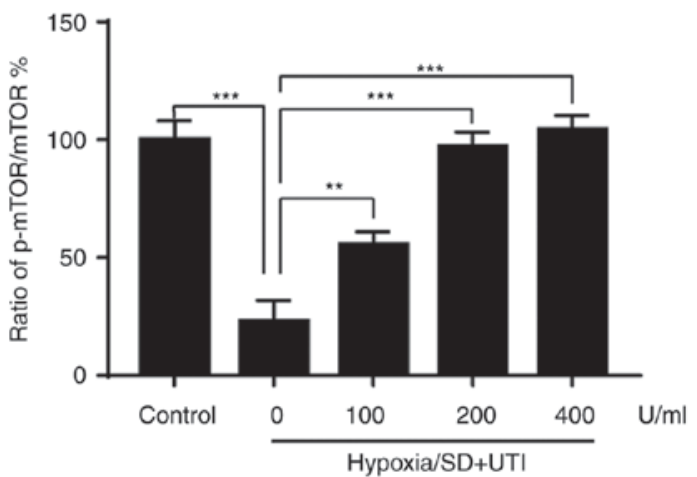

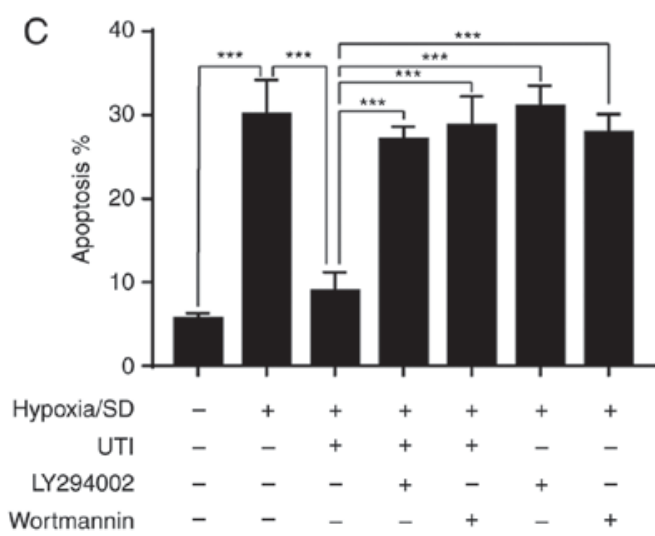

Figure 5. UTI exerts anti-apoptotic effects via activation of the PI3K/Akt pathway. (A) MSCs were treated with various doses of UTI under hypoxia/SD conditions for $12 \mathrm{~h}$. Total cellular lysates were subjected to western blot analysis with the indicated antibodies, and the results were semi-quantified. (B) MSCs were exposed to hypoxia/SD conditions. In parallel experiments, cells were treated with either UTI (400 U/ml), wortmannin (20 $\mu \mathrm{M})$ or LY294002 (20 $\mu \mathrm{M})$. Total cellular lysates were subjected to western blot analysis with the indicated antibodies, and the results were quantified. (C) Cells were treated as above, and then cellular apoptosis was measured. 


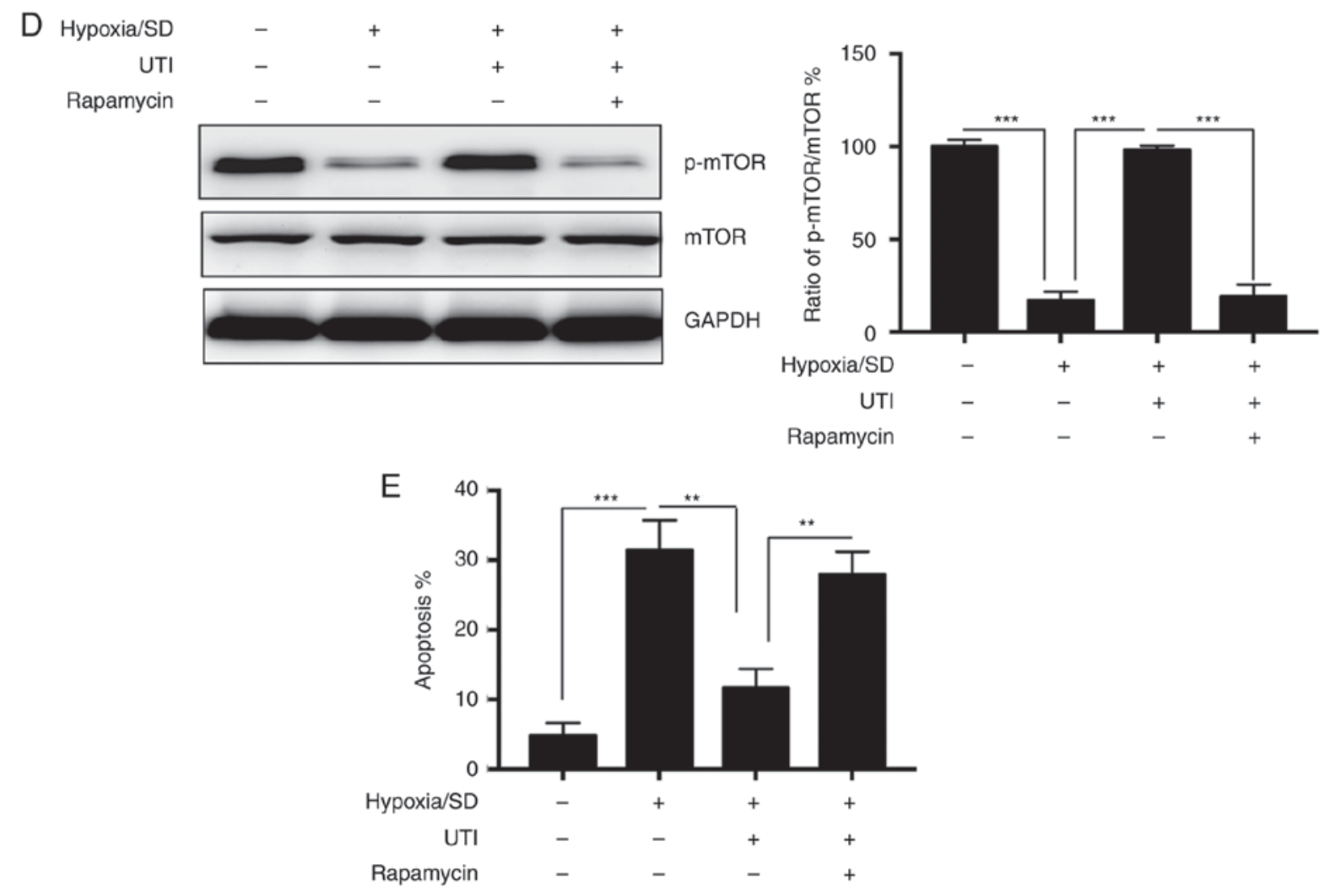

Figure 5. Continued. (D) MSCs were exposed to hypoxia/SD conditions and were treated with either UTI (400 U/ml) or UTI (400 U/ml) plus rapamycin (20 $\mu \mathrm{M})$. Total cellular lysates were subjected to western blot analysis with the indicated antibodies, and the results were quantified. (E) Cells were treated as above, and then cellular apoptosis was measured. The data are presented as the mean \pm standard deviation $(\mathrm{n}=3) .{ }^{* *} \mathrm{P}<0.01 ;{ }^{* * * *} \mathrm{P}<0.001$. MSC, mesenchymal stem cell; SD, serum deprivation; UTI, ulinastatin; p, phosphorylated; PI3K, phosphatidylinositol-4,5-bisphosphate 3-kinase; mTOR, mammalian target of rapamycin.

role in the apoptosis of various types of cells in models of ischemia in vivo and in vitro (26). Furthermore, MSCs are susceptible to apoptosis induced by ER stress, and blockage of ER stress promoted the survival of MSCs under H/SD conditions (27). In response to ER stress, there is an upregulation of ER chaperones such as CHOP and ATF4 (28). In the present study, CHOP and ATF4 expression levels were upregulated under H/SD conditions and treatment with UTI could reverse this effect. These findings indicated that the UTI inhibition of apoptosis of MSCs under H/SD may be attributed to the blockage of ER stress.

$\mathrm{PI} 3 \mathrm{~K} / \mathrm{Akt} / \mathrm{mTOR}$ is a vital cell survival pathway, and Akt phosphorylation enhances survival in various cell types (29). Previous studies have reported that activation of the Akt signaling pathway promoted the survival of MSCs following transplantation into the ischemic porcine heart (30). UTI has been shown to inhibit apoptosis of cardiomyocytes via activation of the PI3K/Akt signaling pathway, which serves a significant protective role against MSC apoptosis (31). The present results demonstrated that UTI activated the $\mathrm{PI} 3 \mathrm{~K} / \mathrm{Akt} / \mathrm{mTOR}$ signaling pathway and that these protective effects were clearly diminished by two PI3K inhibitors, wortmannin and LY294002, and the mTOR inhibitor, rapamycin. These findings suggest a critical role of the PI3K/Akt/mTOR signaling pathway in the UTI-mediated protection of MSCs exposed to H/SD. These results agree with previous studies suggesting that activation of PI3K/Akt/mTOR enhances the survival of MSCs under H/SD conditions (32). Currently, inhibition of the PI3K/Akt/mTOR pathway is used as a therapeutic strategy against various diseases, including human immunodeficiency virus and cancer (33-36). The inhibition of PI3K/Akt/mTOR signaling resulted in increased apoptosis of MSCs. Therefore, the application of PI3K/Akt/mTOR inhibitors to MSCs should be investigated further.

The present study has certain limitations. The H/SD condition is an in vitro model that cannot fully mimic the in vivo microenvironment. Further investigations are required to unveil the cellular mechanisms involved in vitro and in vivo. Additionally, UTI is a well-known regulator of inflammatory processes that may also participate in the survival of MSCs under H/SD conditions. Therefore, the role of inflammasomes in MSC survival and its association with UTI needs to be investigated.

Taken together, the present results provide preliminary evidence indicating that UTI promotes MSC survival under $\mathrm{H} / \mathrm{SD}$ conditions. The pro-survival effects of UTI against $\mathrm{H} / \mathrm{SD}$-induced apoptosis possibly relies on the modulation of the Bcl-2 family of proteins, ER stress responses and PI3K/Akt signaling pathways. Therefore, UTI may be a therapeutic agent with the potential to optimize MSC therapy.

\section{Acknowledgements}

The authors would like to thank Dr Rui Yu (School of Medicine, Ningbo University, Ningbo, Zhejiang, China) for their critical comments on this manuscript. 


\section{Funding}

The present study was supported by grants from the Ningbo Natural Science Foundation of China (Grant no. 2015A610203).

\section{Availability of data and materials}

The datasets used and/or analyzed during the present study are available from the corresponding author on reasonable request.

\section{Authors' contributions}

LS and ZD designed the research. LS, LY, PL and GY performed the experiments. JC and DL analyzed the data. LS wrote the manuscript. LS and ZD revised the manuscript.

\section{Ethics approval and consent to participate}

All studies were performed under the approval of the Institutional Animal Care and Use Committee of Ningbo Medical Center Lihuili Eastern Hospital.

\section{Patient consent for publication}

Not applicable.

\section{Competing interests}

The authors declare that they have no competing interests.

\section{References}

1. Lopez AD, Mathers CD, Ezzati M, Jamison DT and Murray CJ: Global and regional burden of disease and risk factors, 2001 Systematic analysis of population health data. Lancet 367 $1747-1757,2006$

2. Strauer BE and Steinhoff G: 10 years of intracoronary and intramyocardial bone marrow stem cell therapy of the heart: From the methodological origin to clinical practice. J Am Coll Cardiol 58 1095-1104, 2011.

3. Menasché P: Current status and future prospects for cell transplantation to prevent congestive heart failure. Semin Thorac Cardiovasc Surg 20: 131-137, 2008.

4. Zhu W, Chen J, Cong X, Hu S and Chen X: Hypoxia and serum deprivation-induced apoptosis in mesenchymal stem cells. Stem Cells 24: 416-425, 2006.

5. Li C, Ma D, Chen M, Zhang L, Zhang L, Zhang J, Qu X and Wang C: Ulinastatin attenuates LPS-induced human endothelia cells oxidative damage through suppressing JNK/c-Jun signaling pathway. Biochem Biophys Res Commun 474: 572-578, 2016.

6. Liu B, Huang W, Xiao X, Xu Y, Ma S and Xia Z: Neuroprotective effect of ulinastatin on spinal cord ischemia-reperfusion injury in rabbits. Oxid Med Cell Longev 2015: 624819, 2015.

7. Yu Z, Rayile A, Zhang X, Li Y and Zhao Q: Ulinastatin protects against lipopolysaccharide-induced cardiac microvascular endothelial cell dysfunction via downregulation of lncRNA MALAT1 and EZH2 in sepsis. Int J Mol Med 39: 1269-1276, 2017.

8. Makino S, Fukuda K, Miyoshi S, Konishi F, Kodama H, Pan J, Sano M, Takahashi T, Hori S, Abe H, et al: Cardiomyocytes can be generated from marrow stromal cells in vitro. J Clin Invest 103: 697-705, 1999.

9. Yu R, Yu BX, Chen JF, Lv XY, Yan ZJ, Cheng Y and Ma Q: Anti-tumor effects of Atractylenolide I on bladder cancer cells J Exp Clin Cancer Res 35: 40, 2016.

10. Garner TP, Lopez A, Reyna DE, Spitz AZ and Gavathiotis E: Progress in targeting the BCL-2 family of proteins. Curr Opin Chem Biol 39: 133-142, 2017.

11. Maekawa $\mathrm{H}$ and Inagi R: Stress signal network between hypoxia and ER Stress in chronic kidney disease. Front Physiol 8: 74, 2017.
12. Shake JG, Gruber PJ, Baumgartner WA, Senechal G, Meyers J, Redmond JM, Pittenger MF and Martin BJ: Mesenchymal stem cell implantation in a swine myocardial infarct model: Engraftment and functional effects. Ann Thorac Surg 73: 1919-1926, 2002.

13. Orlic D, Kajstura J, Chimenti S, Limana F, Jakoniuk I, Quaini F, Nadal-Ginard B, Bodine DM, Leri A and Anversa P: Mobilized bone marrow cells repair the infarcted heart, improving function and survival. Proc Natl Acad Sci USA 98: 10344-10349, 2001.

14. Marofi F, Vahedi G, Biglari A, Esmaeilzadeh A and Athari SS: Mesenchymal stromal/stem cells: A new era in the cell-based targeted gene therapy of cancer. Front Immunol 8: 1770, 2017

15. Hale SL, Dai W, Dow JS and Kloner RA: Mesenchymal stem cell administration at coronary artery reperfusion in the rat by two delivery routes: A quantitative assessment. Life Sci 83: 511-515, 2008.

16. Hodgetts SI, Beilharz MW, Scalzo AA and Grounds MD: Why do cultured transplanted myoblasts die in vivo? DNA quantification shows enhanced survival of donor male myoblasts in host mice depleted of CD4+ and CD8+ cells or Nk1.1+ cells. Cell Transplant 9: 489-502, 2000.

17. Tang YL, Tang Y, Zhang YC, Qian K, Shen L and Phillips MI: Improved graft mesenchymal stem cell survival in ischemic heart with a hypoxia-regulated heme oxygenase-1 vector. J Am Coll Cardiol 46: 1339-1350, 2005.

18. Zeng K, Deng BP, Jiang HQ, Wang M, Hua P, Zhang HW, Deng YB and Yang YQ: Prostaglandin $\mathrm{E}_{1}$ protects bone marrow-derived mesenchymal stem cells against serum deprivation-induced apoptosis. Mol Med Rep 12: 5723-5729, 2015.

19. Zhang F, Cui J, Lv B and Yu B: Nicorandil protects mesenchymal stem cells against hypoxia and serum deprivation-induced apoptosis. Int J Mol Med 36: 415-423, 2015.

20. Inoue $\mathrm{K}$ and Takano $\mathrm{H}$ : Urinary trypsin inhibitor as a therapeutic option for endotoxin-related inflammatory disorders. Expert Opin Investig Drugs 19: 513-520, 2010.

21. Inoue K, Takano H, Yanagisawa R and Yoshikawa T: Protective effects of urinary trypsin inhibitor on systemic inflammatory response induced by lipopolysaccharide. J Clin Biochem Nutr 43: 139-142, 2008.

22. Atal SS and Atal S: Ulinastatin-a newer potential therapeutic option for multiple organ dysfunction syndrome. J Basic Clin Physiol Pharmacol 27: 91-99, 2016.

23. Bialik S, Cryns VL, Drincic A, Miyata S, Wollowick AL, Srinivasan A and Kitsis RN: The mitochondrial apoptotic pathway is activated by serum and glucose deprivation in cardiac myocytes. Circ Res 85: 403-414, 1999.

24. Chen CC, Liu ZM, Wang HH, He W, Wang Y and Wu WD: Effects of ulinastatin on renal ischemia-reperfusion injury in rats. Acta Pharmacol Sin 25: 1334-1340, 2004.

25. Merksamer PI, Trusina A and Papa FR: Real-time redox measurements during endoplasmic reticulum stress reveal interlinked protein folding functions. Cell 135: 933-947, 2008.

26. Badiola N,Penas C,Miñano-Molina A,Barneda-ZahoneroB,FadóR, Sánchez-Opazo G, Comella JX, Sabriá J, Zhu C, Blomgren K, et al: Induction of ER stress in response to oxygen-glucose deprivation of cortical cultures involves the activation of the PERK and IRE-1 pathways and of caspase-12. Cell Death Dis 2: e149, 2011.

27. Motegi SI, Sekiguchi A, Uchiyama A, Uehara A, Fujiwara C, Yamazaki S, Perera B, Nakamura H, Ogino S, Yokoyama Y, et al: Protective effect of mesenchymal stem cells on the pressure ulcer formation by the regulation of oxidative and endoplasmic reticulum stress. Sci Rep 7: 17186, 2017.

28. Sears TK and Angelastro JM: The transcription factor ATF5: Role in cellular differentiation, stress responses, and cancer. Oncotarget 8: 84595-84609, 2017.

29. Franke TF, Kaplan DR and Cantley LC: PI3K: Downstream AKTion blocks apoptosis. Cell 88: 435-437, 1997.

30. Lim SY, Kim YS, Ahn Y, Jeong MH, Hong MH, Joo SY, Nam KI, Cho JG, Kang PM and Park JC: The effects of mesenchymal stem cells transduced with Akt in a porcine myocardial infarction model. Cardiovasc Res 70: 530-542, 2006.

31. Chen J, Crawford R, Chen C and Xiao Y: The key regulatory roles of the PI3K/Akt signaling pathway in the functionalities of mesenchymal stem cells and applications in tissue regeneration. Tissue Eng Part B Rev 19: 516-528, 2013.

32. Guo Z, Li CS, Wang CM, Xie YJ and Wang AL: CSE/H2S system protects mesenchymal stem cells from hypoxia and serum deprivation-induced apoptosis via mitochondrial injury, endoplasmic reticulum stress and PI3K/Akt activation pathways. Mol Med Rep 12: 2128-2134, 2015. 
33. Maksimovic-Ivanic D, Fagone P, McCubrey Y, Bendtzen K, Mijatovic S and Nicoletti F: HIV-protease inhibitors for the treatment of cancer: Repositioning HIV protease inhibitors while developing more potent $\mathrm{NO}$-hybridized derivatives? Int J Cancer 140: 1713-1726, 2017

34. Nicoletti F, Fagone P, Meroni P, McCubrey J and Bendtzen K: mTOR as a multifunctional therapeutic target in HIV infection. Drug Discovery Today 16: 715-721, 2017.
35. Donia M, McCubrey JA, Bendtzen K and Nicoletti F: Potential use of rapamycin in HIV infection. Br J Clin Pharmacol 70: 784-793, 2010

36. Yu Y, Yu X, Ma J, Tong Y and Yao J: Effects of NVP-BEZ235 on the proliferation, migration, apoptosis and autophagy in HT-29 humancolorectal adenocarcinoma cells. Int J Oncol 49: 285-293, 2016. 